\title{
Experience of a tertiary referral center in managing bladder cancer in conjunction with neurogenic bladder
}

\author{
Gianluca Sampogna ${ }^{1,2} \cdot$ Matteo Maltagliati ${ }^{3} \cdot$ Antonio Galfano $^{4} \cdot$ Aldo Bocciardi $^{4} \cdot$ Bernardo Rocco $^{3}$. \\ Salvatore Micali ${ }^{3} \cdot$ Emanuele Montanari $^{1} \cdot$ Michele Spinelli $^{2}$
}

Received: 17 February 2020 / Revised: 15 June 2020 / Accepted: 16 June 2020

(c) The Author(s), under exclusive licence to International Spinal Cord Society 2020

\begin{abstract}
Study design Case series.

Objectives The aim of this study was to present our experience with the management of bladder cancer (BCa) in individuals followed for neurogenic bladder (NB).

Setting An Italian tertiary referral center for NB.

Methods We retrospectively collected all pre-operative, intra-operative, and post-operative data of our NB cases with BCa, diagnosed from 2004 to 2019.

Results We included ten cases: eight with acquired spinal cord injury (SCI) and two with myelomeningocele (MMC). Considering individuals with acquired SCI, the median age at BCa diagnosis and time since SCI were 53 and 34 years, respectively. One out of seven cases had positive urine cytology. All cases underwent a radical cystectomy, diagnosing squamous cell carcinoma (SCC) and transitional cell carcinoma in 60 and $40 \%$ cases, respectively. Surgical-related complications occurred after $90 \%$ procedures. Three out of eight individuals with acquired SCI died 2, 12, and 80 months after the diagnosis. Both individuals with MMC presented no evidence of disease after 24 and 27 months.

Conclusions $\mathrm{BCa}$ in individuals with NB proved to be associated with a diagnosis at an advanced stage and a high rate of surgical complications. In this population we advocate annual genitourinary ultrasound exam and urine cytology, and cystoscopy in all cases of macrohematuria. Considering the low accuracy of urine cytology and the difficult-to-interpret inflamed bladder walls at cystoscopy in NB, a patient-tailored follow-up schedule based on specific risk factors (e.g., smoking status, indwelling urinary catheter) is mandatory to diagnose and treat $\mathrm{BCa}$ at an early stage.
\end{abstract}

\section{Introduction}

Bladder cancer $(\mathrm{BCa})$ is the 5 th most commonly diagnosed cancer in men worldwide, and the 17 th most common

Michele Spinelli

michele.spinelli@ospedaleniguarda.it

1 Urology Unit, Fondazione IRCCS Ca' Granda, Ospedale Maggiore Policlinico, Università degli Studi di Milano, Milano, MI, Italy

2 Neurourology Service, Unipolar Spinal Unit, ASST Grande Ospedale Metropolitano Niguarda, Milano, MI, Italy

3 Urology Unit, Ospedale Policlinico e Nuovo Ospedale Civile Sant’Agostino-Estense, Università degli Studi di Modena e Reggio Emilia, Baggiovara, MO, Italy

4 Urology Unit, ASST Grande Ospedale Metropolitano Niguarda, Milano, MI, Italy cancer in women [1]. Many risk factors for $\mathrm{BCa}$ have been recognized over the years. The most well-established risk factor is represented by tobacco smoking, which is associated with $50-65 \%$ of all male cases, and with $20-30 \%$ of females [2]. The second most important risk factor for $\mathrm{BCa}$ is represented by the occupational exposure to aromatic amines, polycyclic aromatic hydrocarbons, and chlorinated hydrocarbons, accounting for about $10 \%$ of all cases [3]. Other risk factors are bladder schistosomiasis and recurrent urinary tract infections (rUTIs), which may cause longlasting irritation of the bladder epithelium, thus increasing the $\mathrm{BCa}$ risk [4].

Chronic bladder inflammation is common in people with neurogenic bladder (NB), affected by a variety of diseases altering the nervous control of the urinary tract, e.g., spinal cord injury (SCI), myelomeningocele (MM), and multiple sclerosis [5]. These individuals may perform clean intermittent catheterizations (CICs) or have an indwelling 
urinary catheter (IDC) [6]. The use of an IDC, especially for more than 10 years, is considered a risk factor for $\mathrm{BCa}$ [7]. In addition, individuals with NB often present well-known bladder irritants, like bladder stones and/or rUTIs [8]. This population presents unique challenges and a peculiar disease pattern, characterized by younger age at diagnosis, high proportion of squamous cell carcinoma (SCC), and aggressive behavior [9]. Indeed, a meta-analysis by GuiZhong and Li-Bo [10], including 18 studies about $\mathrm{BCa}$ in SCI, outlined a mean age at diagnosis of 50 years, an estimated percent of SCC of $36.8 \%$, and a 1-year overall survival rate after treatment of $62.1 \%$.

All these issues highlight the importance of a proper follow-up strategy for an early diagnosis, even if there is no current consensus around the world [11].

The aim of this study was to present our experience with the management of $\mathrm{BCa}$ in individuals with NB followed by a tertiary referral center in order to highlight the peculiarities that should be considered for the diagnosis, treatment, and prognosis of $\mathrm{BCa}$ in this population.

\section{Methods}

We considered all individuals with SCI who attended our tertiary referral center for NB-related problems and received a BCa diagnosis from January 2004 to December 2019. We retrospectively collected all case details and clinical outcomes in accordance with our privacy law and with the Declaration of Helsinki.

The pre-operative data included demographic variables (e.g., age at $\mathrm{BCa}$ diagnosis, sex) and details from medical history, in particular smoking status, SCI etiology, years from the index event in case of acquired SCI, previous genitourinary (GU) surgery, bladder management, and history of rUTIs.

We screened for events leading to the $\mathrm{BCa}$ diagnosis, histology after transurethral resection of bladder tumor (TURBT), urinary diversions performed in case of radical cystectomy (RC), complications associated with RC (reported according to the Clavien-Dindo classification system), histology and staging after RC [12].

We assessed the disease status at the last follow-up visit [13]. In case of death, we investigated the cause of death and estimated the time lapse from $\mathrm{BCa}$ diagnosis to death.

Data were stored anonymously and analyzed using Microsoft Excel (Microsoft Corporation, Redmond, WA, USA). We estimated median and interquartile range (IQR) for each quantitative variable, while we reported the occurrence of the various outcomes for qualitative variables. We performed survival analysis with a Kaplan-Meier model, realized with SPSS, version 20 (IBM Corporation, Armonk, NY, USA). We did not include individuals with congenital SCI for descriptive statistics when the early injury timing might alter some specific variables, like age at $\mathrm{BCa}$ diagnosis and prognosis.

\section{Results}

We included ten cases (Table 1), affected by acquired SCI $(n=8)$ and congenital MMC $(n=2)$. Among individuals with acquired SCI, seven cases had traumatic tetraplegia $(n=1)$ and paraplegia $(n=6)$, while one had transverse myelitis $(n=1)$. Eight out of ten individuals were males $(80 \%)$, while $2(20 \%)$ cases were females (both with MMC). In case of acquired SCI, the median age at $\mathrm{BCa}$ diagnosis was 53 years (IQR: 48.75-59; range: 47-83), while the median time lapse from the index event was 34 years (IQR: $28.5-42.5$; range: $22-51$ ). Up to $50 \%$ individuals were smokers at diagnosis.

Five out of ten cases performed CIC (Table 2), while three cases had an IDC, substituted periodically. Five out of ten individuals took muscarinic receptor antagonists (MRA). We identified reflex voiding in two cases.

As for previous GU surgery, 5/10 (50\%) individuals underwent different urethral surgical procedures. Two cases underwent transurethral cystolithotripsy, while three individuals received periodical botulinum toxin injections into the detrusor muscle. Most cases (80\%) reported rUTIs.

Macrohematuria was the presenting condition in all individuals (Table 3). Urine cytology was positive in one out of seven cases, while negative in $85.7 \%$ individuals.

Nine out of ten cases $(90 \%)$ underwent TURBT with the following diagnosis: transitional cell carcinoma (TCC) in $6 /$ $9(66.7 \%)$ individuals, with carcinoma in situ in $2 / 9$ cases, and SCC in the other 3/9 individuals (33.3\%).

We addressed 10/10 cases to RC (Fig. 1). We performed Bricker's ileal conduits and ureterocutaneostomies in 60 and $30 \%$ individuals, respectively. One man underwent an orthotopic ileal neobladder. After RC, the histology revealed predominantly a SCC and TCC in 60 and 40\% cases, respectively. Staging was the following: T1 $(n=1)$, Tis $(n=1)$, T2 $(n=5)$, T3 $(n=1)$, and T4 $(n=2)$. No intra-operative surgical complications occurred. Nine out of ten $(90 \%)$ cases reported different-graded post-operative complications: grade I $(n=2,20 \%)$, grade II $(n=4,40 \%)$, grade III $(n=3,30 \%)$.

In Table 4, we reported the follow-up of our study population. In case of acquired SCI, the median overall survival was 80 months; $3 / 8$ individuals died for cancerspecific complications 2,12 , and 80 months after the $\mathrm{BCa}$ diagnosis (Fig. 2). Both women with MMC were alive at the last follow-up visit 24 and 27 months after the $\mathrm{BCa}$ diagnosis. We highlighted no evidence of disease in all $7 / 10$ (70\%) living individuals. 
Table 1 Demographic characteristics of the enrolled population.
Table 2 Evaluation of bladder management, previous genitourinary surgery, and recurrent urinary tract infections in the enrolled population.

\begin{tabular}{llllll}
\hline ID & SCI etiology & Sex & $\begin{array}{l}\text { Age at BCa } \\
\text { diagnosis (years) }\end{array}$ & $\begin{array}{l}\text { Time since SCI* } \\
\text { (years) }\end{array}$ & $\begin{array}{l}\text { Smoker at } \\
\text { diagnosis }\end{array}$ \\
\hline 1 & Congenital MMC & F & 32 & - & No \\
2 & Congenital MMC & F & 58 & - & Yes \\
3 & TM & M & 83 & 40 & No \\
4 & Traumatic SCI C7 & M & 71 & 51 & No \\
5 & Traumatic SCI D4 & M & 48 & 27 & No \\
6 & Traumatic SCI D4-D5 & M & 53 & 37 & Yes \\
7 & Traumatic SCI D12 & M & 53 & 22 & Yes \\
8 & Traumatic SCI L1-L2 & M & 55 & 50 & No \\
9 & Traumatic SCI L1-L2 & M & 49 & 31 & Yes \\
10 & Traumatic SCI D3 & M & 47 & 29 & Yes \\
Cases: 10 & MMC: $2 / 10$ (20\%) & F: 2/10 (20\%) & Median*: 53 & Median: 34 & No: 5/10 (50\%) \\
& TM: $1 / 10(10 \%)$ & M: $8 / 10(80 \%)$ & IQR*: 48.75-59 & IQR: 28.5-42.5 & Yes: 5/10 (50\%) \\
& Traumatic SCI: 7/10 (70\%) & & Range*: 47-83 & Range: $22-51$ & \\
\hline
\end{tabular}

$B C a$ bladder cancer, $F$ female, $I Q R$ inter-quartile range, $M$ male, $M M C$ myelomeningocele, $S C I$ spinal cord injury, $T M$ transverse myelitis.

*Data estimated considering only individuals with acquired spinal cord injury.

\begin{tabular}{llll}
\hline ID & Bladder management & History of GU surgeries & rUTIs \\
\hline 1 & CIC + MRA & $\begin{array}{l}\text { Urethral bulking BTX injections into the } \\
\text { detrusor wall }\end{array}$ & No \\
2 & CIC & No & Yes \\
3 & IDC & TUCL & Yes \\
4 & IDC + MRA & Urethral fistulectomies & Yes \\
5 & Reflex voiding & No & Yes \\
6 & CIC + MRA & Endoscopic urethrotomy TUCL & No \\
7 & CIC + MRA & Urethral fistulectomies BTX injections into the & Yes \\
8 & Reflex voiding & Ureterocelectomy Urethroplasty & Yes \\
9 & IDC & No & Yes \\
10 & CIC + MRA & BTX injections into the detrusor wall & Yes \\
Cases: 10 & CIC: $5 / 10(50 \%)$ & Urethral surgery: $5 / 10$ (50\%) & No: $2 / 10(20 \%)$ \\
& IDC: $3 / 10(30 \%)$ & BTX injections into the detrusor wall: $3 / 10(30 \%)$ & Yes: $8 / 10(80 \%)$ \\
& MRA: $5 / 10(50 \%)$ & TUCL: $2 / 10(20 \%)$ &
\end{tabular}

$B T X$ botulinum toxin, $C I C$ clean intermittent catheterization, $G U$ genitourinary, $I D C$ indwelling urinary catheter, MRA muscarinic receptor antagonist, rUTIs recurrent urinary tract infections, $R V$ reflex voiding, TUCL transurethral cystolithotripsy.

\section{Discussion}

The incidence and prevalence of NB are increasing worldwide [14]. To start with, individuals with SCI are now living longer with an improved quality of life thanks to medical advances [15]. Considering long-term complications, $\mathrm{BCa}$ is a life-threating disease which deserves an early diagnosis and treatment [16]. Indeed, $\mathrm{BCa}$ is the third most frequent cause of death among individuals with SCI [17].
People with NB may present several added risk factors for BCa: tobacco smoking, chronic inflammation of the bladder secondary to the presence of IDCs, bladder stones, and rUTIs [18]. In our study, rUTIs, smoking, and the use of IDC were identified in 80,50 , and $30 \%$ cases, respectively. Five out of ten individuals had previously undergone urethral surgery, which may be associated with metaplasia, thus increasing the risk of $\mathrm{BCa}$, in particular SCC. The relevance of this correlation has not been fully acknowledged in the literature and further larger, long-term studies are mandatory to understand the relationship [19]. Chronic inflammation is usually associated with SCC, which was the 


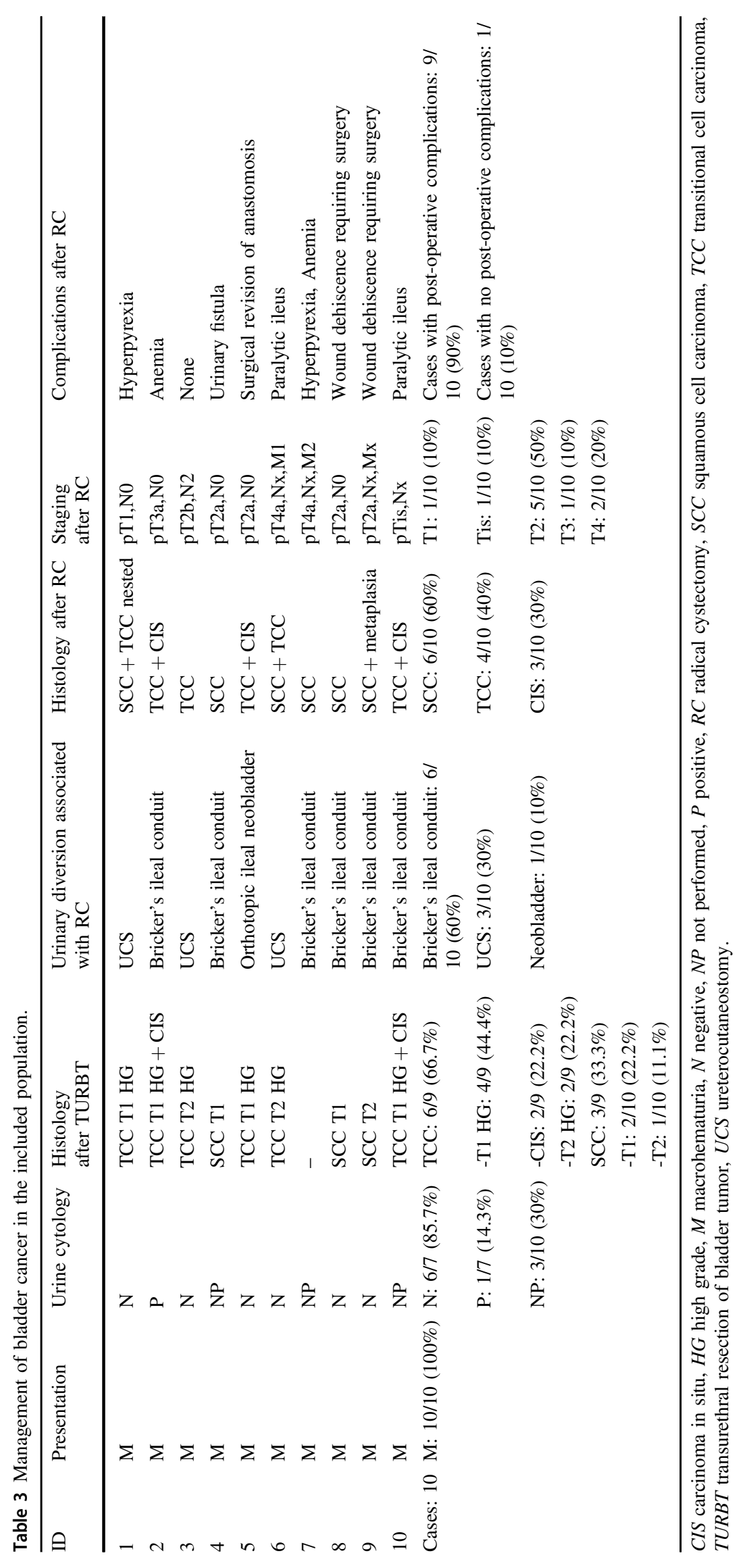




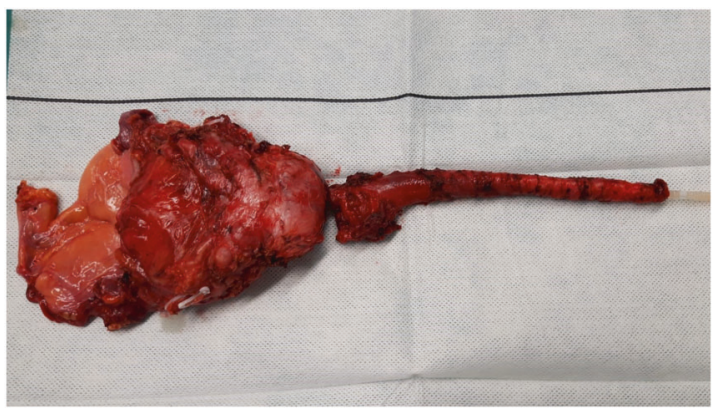

Fig. 1 Surgical specimen of case $n^{\circ} 10$. The patient underwent a laparoscopic radical cystectomy and perineal urethrectomy, then an intracorporeal Bricker's ileal conduit urinary diversion was realized. Surgical pathology revealed pTis, pNx.

Table 4 Follow-up of the considered population.

\begin{tabular}{lll}
\hline ID & $\begin{array}{l}\text { Months from BCa diagnosis to last } \\
\text { follow-up visit or death }\end{array}$ & Status \\
\hline 1 & 27 & NED \\
2 & 24 & NED \\
3 & 195 & NED \\
4 & 80 & CSD \\
5 & 60 & NED \\
6 & 12 & CSD \\
7 & 2 & CSD \\
8 & 41 & NED \\
9 & 7 & NED \\
10 & 3 & NED \\
Cases: 10 & Median overall survival: $80^{*}$ & CSD: $3 /$ \\
& & $10(30 \%)$ \\
& & NED: $7 /$ \\
& & $10(70 \%)$
\end{tabular}

$B C a$ bladder cancer, $C S D$ cancer-specific death, $N E D$ no evidence of disease.

*Data estimated considering only individuals with acquired spinal cord injury.

most common histology in our series $(60 \%)$, even if other similar case series reported TCC as the most frequent histotype [20, 21]. Besides associated risk factors, current tendency is to consider NB itself a condition favoring $\mathrm{BCa}$ development [10].

SCI may be associated with a significantly increased incidence of $\mathrm{BCa}$ that has been reported as up to 16-28 times higher compared to the general population [22]. These individuals present unique challenges. Since they usually perform CICs or have an IDC, macrohematuria is common, frequently due to improper maneuvers and/or rUTIs. Therefore, this issue may divert the attention from the $\mathrm{BCa}$, especially at an early stage when macrohematuria is often minimal and sporadic [23]. All our cases were diagnosed

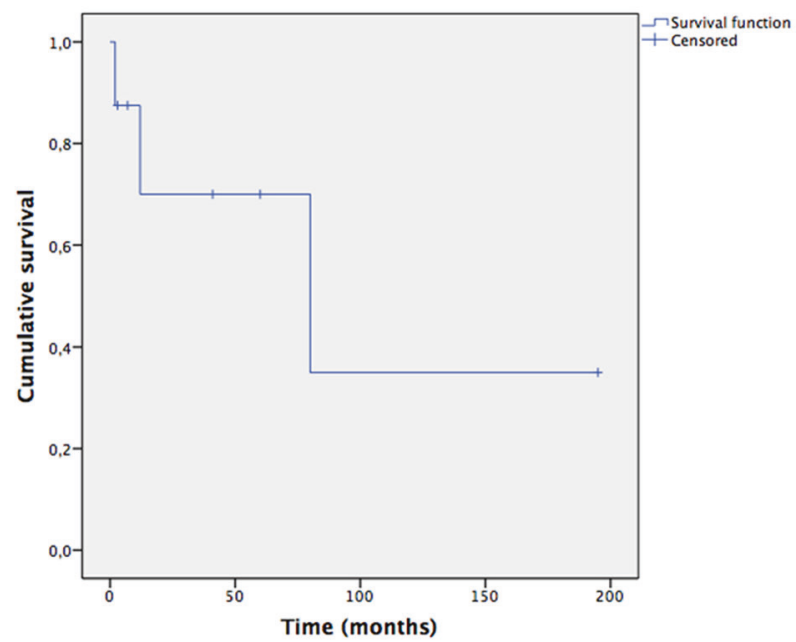

Fig. 2 Survival curve in case of acquired spinal cord injury. Kaplan-Meier plot displaying the survival of eight cases after the bladder cancer diagnosis. Three individuals died after 2, 12, and 80 months.

because of long-term macrohematuria, initially underestimated.

Among symptoms in individuals with SCI, multiple episodes of autonomic dysreflexia (AD) may be associated with locally invasive $\mathrm{BCa}$, requiring $\mathrm{RC}$ to alleviate symptoms [24]. A comprehensive diagnostic work-up is mandatory in individuals with SCI with a lesion above T6, who present recurrent $\mathrm{AD}$, as it may be the only presenting symptom of life-threating underlying diseases, like $\mathrm{BCa}$ [25].

Our study highlighted many cases of $\mathrm{BCa}$ diagnosed at an advanced stage, associated with a poor prognosis. Indeed, most cases $(80 \%)$ were muscle-invasive forms and 3 out of $10(30 \%)$ individuals died for cancer-specific complications. This was partially due to a delayed diagnosis due to an underestimated macrohematuria [26]. Compared to the general population, the study by Nahm et al. highlighted an increased BCa mortality in individuals with SCI of comparable age, gender, and race, with a standardized mortality ratio (SMR) of 6.69 with a $95 \%$ confidence interval (CI) of 5.44-8.14 [27]. In particular, a time lapse greater than 20 years after SCI was associated with a significantly increased SMR: 17.83 (95\% CI: 14.00-22.39).

The role of urine cytology in the early diagnosis of $\mathrm{BCa}$ in individuals with NB is a debated issue. In our series, one out of seven cases had positive urine cytology. Since the urine cytology has low accuracy in SCC diagnosis and the incidence of SCC seems to be higher in this population, urine cytology may have a reduced role during the followup of people with NB [28]. A systematic review published in 2018 concluded urinary cytology outperformed cystoscopy for $\mathrm{BCa}$ screening: sensitivity and specificity for 
urine cytology vs. cystoscopy were, respectively, 0-72\% and $100 \%$ vs. $27-81 \%$ and $54 \%$ [29].

Inflamed and diverticular walls in NB may be difficult to interpret, undermining diagnostic accuracy, and inducing a high-rate of false positives: these limits may be overcome by novel promising imaging technologies, like photodynamic diagnosis and narrow-band imaging, even if their role has to be still investigated thoroughly in the NB population [30, 31].

Surveillance patterns vary around the world, as there is no consensus among authors and organizations [32]. At our center, we assess our NB cases annually, updating medical history, performing clinical examination, and viewing bladder diary and reports of renal laboratory tests, urinalysis, urine cytology, and imaging surveillance of the urinary tract, usually via GU ultrasound exam [33]. Further study may be required in case of suspicious signs and/or symptoms (i.e., macrohematuria), changes in bladder behavior (i.e., increased urine leaks), or presence of risk factors (i.e., tobacco use) [34]. In these cases, follow-up plans should be modified with more frequent checkups and/or additional diagnostic exams, like cystoscopy, videourodynamics, abdominal computed tomography scan, and renal scintigraphy.

Until more accurate diagnostic strategies are available, we advocate at least annual urine cytology and GU ultrasound exam in asymptomatic cases. The timing of followup may be shortened in case of specific risk factors (e.g., IDC, smoking habit, and occupational exposure) [35]. Considering the high rate of false negatives with urine cytology, bladder cystoscopy with biopsies of the suspicious areas is required in all cases of macrohematuria [36].

We considered individuals with congenital MMC as a different subset for descriptive statistics of baseline characteristics and follow-up, as the associated injury provoked $\mathrm{NB}$ from fetal life and might induce $\mathrm{BCa}$ in a younger stage of life. Indeed, the youngest case at diagnosis (32 years) was affected by MMC. Previous studies identified 15-20 years since the index event as a warning to investigate $\mathrm{BCa}$ in individuals with acquired SCI. Therefore, clinicians should exclude $\mathrm{BCa}$ in all young adults with MMC presenting with macrohematuria, as they developed a NB during fetal life.

The urinary diversion choice in this population should consider manual dexterity, individual preferences, caregiver support, disease stage and residual renal function [37]. Only one case underwent an orthotopic ileal neobladder because of the disease characteristics (tumor distant from the bladder neck), individual's conditions (valid performance status, young age) and surgeon's preferences [38]. In addition, this man did not void by the Valsalva maneuver, but continued CICs.

We registered a very high rate of surgical complications $(90 \%)$ [39]. This was due to the limited functional reserve to cope with surgical stress [40]. The current treatment guidelines for $\mathrm{BCa}$ have been developed for the general population without considering the challenges and needs of this population. Some precautions may be adopted in the future to reduce surgical morbidity. Post-operative anemia may be limited by a pre-operative iron deficiency detection and eventually through intravenous iron formulations [41]. Antibiotic therapy may be started $48 \mathrm{~h}$ before surgery to limit post-operative infections and febrile episodes. Paralytic ileus in neurogenic bowel may be reduced with adequate bowel preparation, pharmacotherapy with pyridostigmine or alvimopan, and adherence to the recommendations from the Enhanced Recovery After Surgery Society [42]. Future randomized controlled trials may evaluate outcome improvements with these proposed solutions.

Considering the high rate of complications following open major surgery, an early diagnosis of $\mathrm{BCa}$ is essential to perform a radical TURBT and reduce the morbidity in this population.

Our study presents several limits. It has a retrospective design; the level of evidence is four according to the Oxford Center for Evidence-based Medicine [43]. The follow-up is short for some cases. The sample is relatively small, even if it includes all individuals with NB followed by our center, so it is representative of the incidence and prevalence of $\mathrm{BCa}$ in people treated by a tertiary referral center for NB. We have noticed an increased incidence of $\mathrm{BCa}$ in the considered population over the last years, probably due to the extended life expectancy of the individuals with SCI.

As for future directions, our study represents a contribution to outline the peculiar BCa disease pattern and the patient-specific conditions for a proper follow-up of people with NB, also by presenting data for further systematic reviews and meta-analyses [44]. Since the prevalence and incidence of individuals with SCI are increasing worldwide, appropriate indications in terms of follow-up to diagnose and treat $\mathrm{BCa}$ at an early stage should be a priority of scientific societies and organizations involved in the management of this population [45].

\section{Conclusions}

We reported the experience of a tertiary referral center in the management of $\mathrm{BCa}$ in individuals followed for NB. BCa in this population proved to be particularly aggressive, usually diagnosed as SCC at an advanced stage, and associated with a high rate of post-operative complications and mortality. Considering the limits of current diagnostic strategies, like urine cytology and cystoscopy, further multi-center studies focusing on long-term management and carcinogenesis in NB are mandatory to define a proper follow-up schedule to diagnose $\mathrm{BCa}$ at an early stage and improve the prognosis and quality of life of these individuals. Until then, we 
advocate a life-long follow-up strategy based on at least annual GU ultrasound exam and urine cytology, a patienttailored approach considering specific risk factors (e.g., smoking habit, long-term IDC), and prompt cystoscopy in all cases of macrohematuria.

\section{Compliance with ethical standards}

Conflict of interest The authors declare that they have no conflict of interest.

Publisher's note Springer Nature remains neutral with regard to jurisdictional claims in published maps and institutional affiliations.

\section{References}

1. Ferlay J, Soerjomataram I, Ervik M, Dikshit R, Eser S, Mathers C, et al. Cancer incidence and mortality worldwide: sources, methods and major patterns in GLOBOCAN 2012. Int J Cancer. 2015;136: E359-86.

2. Freedman N, Silverman D, Hollenbeck A, Schatzkin A, Abnet C. Association between smoking and risk of bladder cancer among men and women. JAMA. 2011;306:737-45.

3. Burger M, Catto J, Dalbagni G, Grossman H, Herr H, Karakiewicz $\mathrm{P}$, et al. Epidemiology and risk factors of urothelial bladder cancer. Eur Urol. 2013;63:234-41.

4. Pelucchi C, Bosetti C, Negri E, Malvezzi M, La Vecchia C. Mechanisms of disease: the epidemiology of bladder cancer. Nat Clin Pr Urol. 2006;3:327-40.

5. Ginsberg D. The epidemiology and pathophysiology of neurogenic bladder. Am J Manage Care. 2013;19:s191-6.

6. El Masriy WS, Patil S, Prasanna KV, Chowdhury JR. To cystoscope or not to cystoscope patients with traumatic spinal cord injuries managed with indwelling urethral or suprapubic catheters? That is the question! Spinal Cord. 2014;52:49-53.

7. Ismail S, Karsenty G, Chartier-Kastler E, Cussenot O, Compérat E, Rouprêt M, et al. Prevalence, management, and prognosis of bladder cancer in patients with neurogenic bladder: a systematic review. Neurourol Urodyn. 2018;37:1386-95.

8. Stillman MD, Hoffman JM, Barber JK, Williams SR, Burns SP. Urinary tract infections and bladder management over the first year after discharge from inpatient rehabilitation. Spinal Cord Ser Cases. 2018;4:92.

9. Mirkin K, Casey JT, Mukherjee S, Kielb SJ. Risk of bladder cancer in patients with spina bifida: Case reports and review of the literature. J Pediatr Rehabil Med. 2013;6:155-62.

10. Gui-Zhong L, Li-Bo M. Bladder cancer in individuals with spinal cord injuries: a meta-analysis. Spinal Cord. 2017;55:341-45.

11. Averbeck MA, Madersbacher H. Follow-up of the neurourological patient: a systematic review. BJU Int. 2015;115:39-46.

12. Clavien PA, Barkun J, de Oliveira ML, Vauthey JN, Dindo D, Schulick RD, et al. The Clavien-Dindo classification of surgical complications. Ann Surg. 2009;250:187-96.

13. Eisenhauer E, Therasse P, Bogaerts J, Schwartz L, Sargent D, Ford $\mathrm{R}$, et al. New response evaluation criteria in solid tumours: revised RECIST guideline (version 1.1). Eur J Cancer. 2009;45:228-47.

14. Böthig R, Kurze I, Fiebag K, Kaufmann A, Schöps W, Kadhum T, et al. Clinical characteristics of bladder cancer in patients with spinal cord injury: the experience from a single centre. Int Urol Nephrol. 2017;49:983-94.

15. Böthig R, Hirschfeld S, Thietje R. Quality of life and urological morbidity in tetraplegics with artificial ventilation managed with suprapubic or intermittent catheterisation. Spinal Cord. 2012;50:247-51.

16. Parra J, Drouin S, Comperat E, Misraï V, Van Glabeke E, Richard $\mathrm{F}$, et al. Bladder cancer in neurological patients: analysis of a single-centre series. Prog Urol. 2007;17:1333-6.

17. Prattley S, New F, Davies M. Malignancies of suprapubic catheter (SPC) tracts in spinal cord injury patients: a case series and review of literature. Spinal Cord Ser Cases. 2019;5:34.

18. Lee WY, Sun LM, Lin CL, Liang JA, Chang YJ, Sung FC, et al. Risk of prostate and bladder cancers in patients with spinal cord injury: a population-based cohort study. Urol Oncol Semin Orig Investig. 2014;32:51.e1-7.

19. Sun Y, Tang C, Bai Y, Li N, Yang L, Wei Q, et al. Urethral diverticular with broadly squamous metaplasia in a patient with urethral diverticular calculi. Medicine. 2019;98:e16923.

20. Ali P, Lefevrec C, Perrouin-Verbec B, Le Normand L, Rigaud J, Bouchotd $\mathrm{O}$, et al. Bladder cancer in neurogenic patients: a retrospective study of management and follow-up. Progrès en Urol. 2017;28:215-20.

21. Manach Q, Cussenot O, Rouprêt M, Gamé X, Chartier-Kastler E, Reus $\mathrm{C}$, et al. Analysis of bladder cancer subtypes in neurogenic bladder tumors. Can J Urol. 2018;25:9161-67.

22. Kalisvaart JF, Katsumi HK, Ronningen LD, Hovey RM. Bladder cancer in spinal cord injury patients. Spinal Cord. 2010;48:257-61.

23. Böthig R, Fiebag K, Kowald B, Hirschfeld S, Thietje R, Kurze I, et al. Spinal cord injury with neurogenic lower urinary tract dysfunction as a potential risk factor for bladder carcinoma. Aktuelle Urol. 2019;50:280-91.

24. Fitzpatrick R, Baverstock R. Muscle invasive bladder cancer presenting as recurrent autonomic dysreflexia- a wolf in wolf's clothing. J Spinal Cord Med. 2018;20:1-4.

25. Davis M. When guidelines conflict: patient safety, quality of life, and CAUTI reduction in patients with spinal cord injury. Spinal Cord Ser Cases. 2019;5:56.

26. Cheng JN, Lawrentschuk N, Gyomber D, Rogerson J, Bolton DM. Cystectomy in patients with spinal cord injury: indications and long-term outcomes. J Urol. 2010;184:92-8.

27. Nahm LS, Chen Y, Devivo MJ, Lloyd LK. Bladder cancer mortality after spinal cord injury over 4 decades. J Urol. 2015;193:1923-8.

28. Elliott SP. Screening for bladder cancer in individuals with spinal cord injury. J Urol. 2015;193:1880-1.

29. Alimi Q, Hascoet J, Manunta A, Kammerer-Jacquet SF, Verhoest G, Brochard C, et al. Reliability of urinary cytology and cystoscopy for the screening and diagnosis of bladder cancer in patients with neurogenic bladder: a systematic review. Neurourol Urodyn. 2018;37:916-25.

30. Schubert T, Rausch S, Fahmy O, Gakis G, Stenzl A. Optical improvements in the diagnosis of bladder cancer: implications for clinical practice. Ther Adv Urol. 2017;9:251-60.

31. Chen C, Hao H, Tianxin L, Jian H. Diagnostic accuracy of image technique based transurethral resection for non-muscle invasive bladder cancer. Eur Urol Suppl. 2019;18:e1956.

32. Przydacz M, Chlosta P, Corcos J. Recommendations for urological follow-up of patients with neurogenic bladder secondary to spinal cord injury. Int Urol Nephrol. 2018;50:1005-16.

33. Cameron AP, Rodriguez GM, Schomer KG. Systematic review of urological followup after spinal cord injury. J Urol. 2012;187:391-7.

34. Biering-Sørensen F, Kennelly M, Kessler TM, Linsenmeyer T, Pannek J, Vogel L, et al. International spinal cord injury lower urinary tract function basic data set (version 2.0). Spinal Cord Ser Cases. 2018;4:60.

35. McColl MA, Gupta S, Smith K, McColl A. Promoting long-term health among people with spinal cord injury: What's new? Int J Environ Res Public Health. 2017;14:1520. 
36. Sammer U, Walter M, Knüpfer SC, Mehnert U, Bode-Lesniewska B, Kessler TM. Do we need surveillance urethro-cystoscopy in patients with neurogenic lower urinary tract dysfunction? PLoS ONE. 2015; 10:e0140970.

37. Maurice M, Meeks J, Smith N. Orthotopic neobladder for bladder cancer and neurogenic bladder dysfunction. Can $\mathrm{J}$ Urol. 2008;15:4194-5.

38. James A, Lin D, Wright J. Neobladders and continent catheterizable stomas for the bladder cancer survivor. Curr Opin Urol. 2014;24:407-14.

39. Novara G, Catto JWF, Wilson T, Annerstedt M, Chan K, Murphy DG, et al. Systematic review and cumulative analysis of perioperative outcomes and complications after robot-assisted radical cystectomy. Eur Urol. 2015;67:376-401.

40. Yates CC, Garrison MK, Charlesworth A, Reese NB, Garcia-Rill E. Therapeutic approaches for spinal cord injury induced spasticity. Transl Neurosci. 2010;1:160-69.
41. Auerbach M, Macdougall I. The available intravenous iron formulations: history, efficacy, and toxicology. Hemodial Int. 2017;21:S83-S92.

42. Nygren J, Thacker J, Carli F, Fearon KCH, Norderval S, Lobo DN, et al. Guidelines for perioperative care in elective rectal/pelvic surgery: enhanced recovery after surgery $\left(\right.$ ERAS $\left.^{\circledast}\right)$ society recommendations. World J Surg. 2013;37:285-305.

43. Burns P, Rohrich R, Chong K. The levels of evidence and their role in evidence-based medicine. Plast Reconstr Surg. 2011;128:305-10.

44. Hollingsworth J, Rogers M, Krein S, Hickner A, Kuhn L, Cheng A, et al. Determining the noninfectious complications of indwelling urethral catheters. Ann Intern Med. 2013;159:401-10.

45. Qu L, Lawrentschuk N. Bladder cancer surveillance in patients with spinal cord injuries. BJU Int. 2019;123:379-80. 